\title{
Spinal Lesionectomy for Progressive Complete Paraplegia with Recurrent Pilocytic Astrocytoma of the Thoracic Spine: A Case Report
}

\begin{abstract}
Dou Young Park, II Choi, II Young Shin
Department of Neurosurgery, Hallym University Dongtan Sacred Heart Hospital, Hallym University College of Medicine, Hwaseong, Republic of Korea

Corresponding author:

Dou Young Park

Department of Neurosurgery,

Hallym University Dongtan

Sacred Heart Hospital, Hallym

University College of Medicine,

7, Keunjaebong-gil, Hwaseong

18450, Republic of Korea

Tel: $+82-31-8086-3000$

Fax: $+82-31-8086-2029$

E-mail: dypark1016@gmail.com

Received: December 2, 2019

Pilocytic astrocytoma is most often a benign and low-grade tumor with a favorable prognosis. This report presents the case of a 24-year-old woman with rapidly progressive weakness of the bilateral lower extremities. The manual motor test was Grade I. She underwent thoracic laminoplasty from level T2 to T8 and a lesionectomy was performed in our department. Despite surgical intervention, the lower extremity weakness deteriorated to Grade 0. Serial magnetic resonance imagings (MRIs) revealed that the tumor mass was recurring and that syrinx expansion was further contributing to the spinal cord compression. Two years later, this patient presented complaining of upper extremity weakness. On the MRI scan, the syrinx was further expanded and cord signal change of the cervical spine was noted. Revision laminoplasty was performed with a massive spinal lesionectomy from T1 to T12 under intraoperative neuro-monitoring. Radiation therapy of the entire spinal cord was performed to prevent further recurrence following surgery.
\end{abstract}

Revised: March 13, 2020

Accepted: April 3, 2020
Key Words: Astrocytoma; Paraplegia; Spine

\section{INTRODUCTION}

Spinal cord tumors are a relatively rare cause of back pain in adult and pediatric patients. These lesions often manifest as back pain radiating to the extremities and neurological deficits. Spinal cord tumors can be classified as extradural or intradural, and tumors in the intradural compartment can be further divided into extramedullary or intramedullary ${ }^{19)}$.

Astrocytomas account for $80 \%$ to $90 \%$ of the intramedullary spinal cord tumors in childhood and approximately 60\% of intramedullary spinal cord tumors in adolescence ${ }^{19)}$.

Pilocytic astrocytomas are described as benign, slow-growing tumors ${ }^{3)}$. They are classified as Grade I tumors by the World Health Organization (WHO) and are described as occurring principally in pediatric patients, most often featuring a benign prognosis. These tumors can occur at all levels of the spinal cord; however, they occur most frequently in the cerebellum and the optic pathway and occasionally within the spinal canal ${ }^{6}$.

Pilocytic astrocytomas also account for approximately 25\% of pediatric and $1.5 \%$ of adult brain tumor patients with an incidence rate of 4.8 per million per year, globally ${ }^{8)}$. The incidence rate is shown to be similar in both sexes. Intramedullary astrocytomas are dominantly located in the cervico- thoracic or thoracic region in the pediatric population but are found to be more commonly located in the cervical region among the adult population ${ }^{19)}$.

In this article, the rapid progression and recurrence of a primary spinal cord pilocytic astrocytoma has been discussed. This case stood out from the usually benign Grade I lesion described by the WHO. The personalized treatment options for this unusually aggressive lesion were discussed and the protocols for the treatment of recurrent pilocytic astrocytomas were considered.

\section{CASE REPORT}

The patient that will be discussed in this case report was well known to the neurological department of the Dongtan Medical Centre as a neurofibromatosis-1 (NF-1) patient. This 24-year-old woman visited the emergency department complaining of rapidly progressive weakness of her bilateral lower limbs. On examination it was determined that the manual motor test of her lower limbs scored a Grade I level. On magnetic resonance imaging (MRI) investigations, it was determined that the patient had a large enhanced mass with a cystic portion from the T2 to T8 level of the thoracic spine. This enhanced MRI scan is shown in Fig. 1. 
This patient underwent laminoplasty of the T2 to T8 area, and tumor mass removal. The pathology report indicated a low grade pilocytic astrocytoma. Despite the surgical intervention, the lower extremity weakness deteriorated further to Grade 0 and a serial MRI revealed that the tumor mass was recurring with syrinx expansion contributing to the deteriorating clinical picture.

Two years later the patient returned with the complaint of recent onset upper extremity weakness. An MRI was performed and demonstrated further expansion of the syrinx was more with cord signal change of cervical spine present.

Pathologic report: Low grade glioma with perivascular hyalinization and focal myxoid stroma, suggestive of pilocytic astrocytoma. This is shown in Fig. 2.

Revision laminoplasty was performed with aggressive spinal lesionectomy from T1 to T12 under intraoperative neuro-monitoring.

This case of progressive neurologic deficit that required extensive resection of the spinal tumor was determined to be recurrent pilocytic astrocytoma of thoracic spine. Discussion of the case with other experts determined that there are many treatment options for recurrent pilocytic astrocytoma in the spinal cord and it was determined that adjuvant radiotherapy of the entire spinal cord should be performed to prevent recurrence of the tumor.
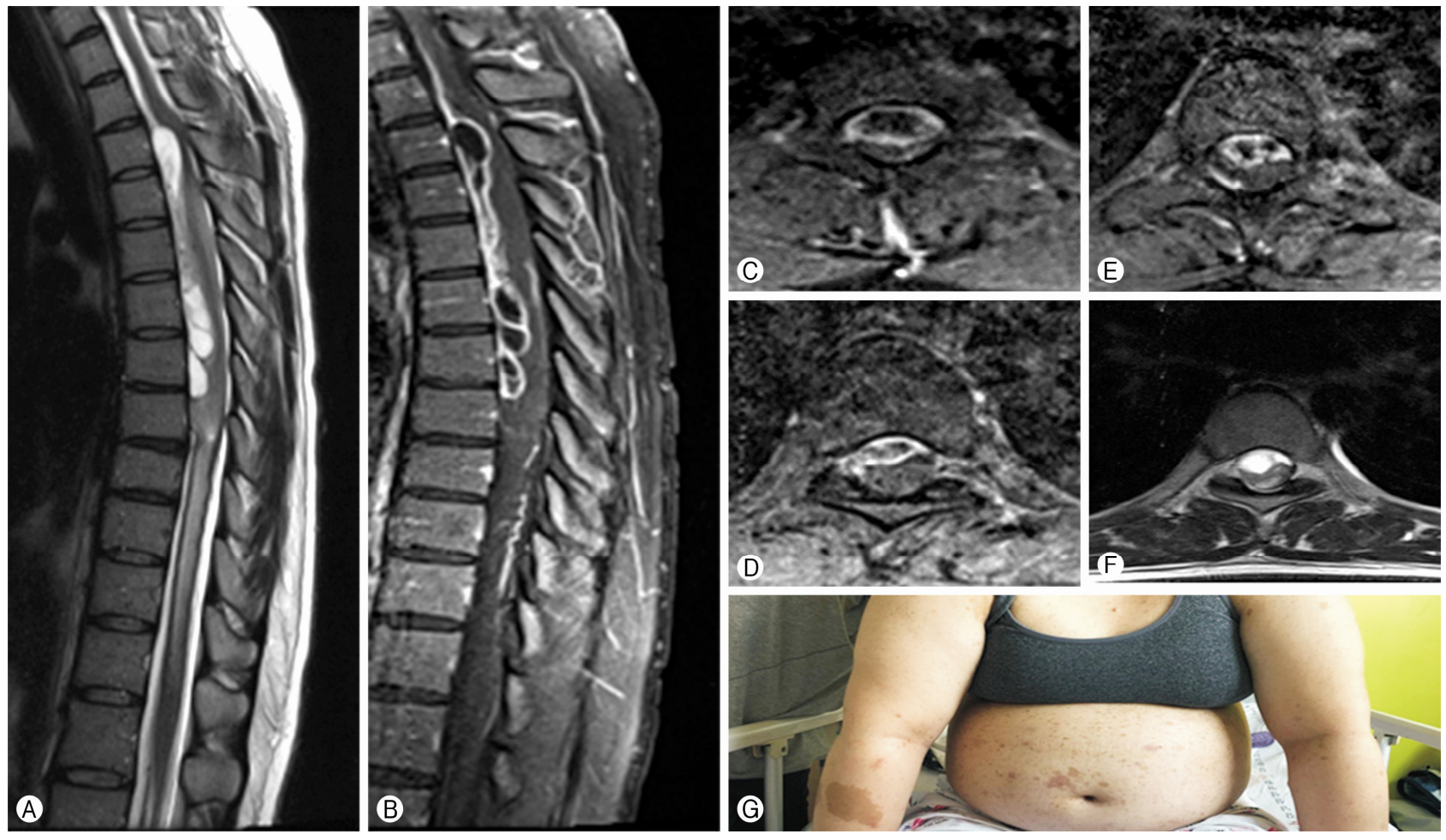

Fig. 1. (A) An elongated, multi-loculated high signal intensity lesion measuring about $10.4 \times 0.9 \times 1.1 \mathrm{~cm}$. (B-E) Magnetic resonance imaging scan show peripheral enhancement at the anterior epidural space from T3 to T8 levels. (F) Posterior displacement and swelling with high signal intensity of the spinal cord are seen from T3 to T8 levels. (G) The patient is a known neurofibromatosis 1 patient with café au lait spots.

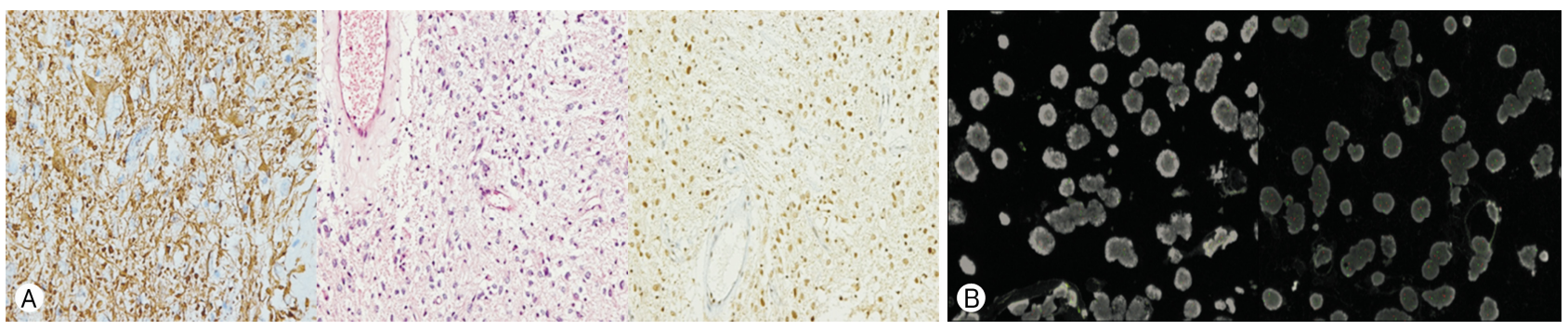

Fig. 2. (A) Low-grade glioma with perivascular hyalinization and focal myxoid stroma, suggestive of pilocytic astrocytoma. (B) Chromosome 1p/19q: no deletion with polysomy is noted. 


\section{DISCUSSION}

The incidence rate of pilocytic astrocytomas, according to the World Standard Population, was 4.8 in 1 million per year. The incidence of pilocytic astrocytomas in children less than 15 years old, when age-adjusted to both the World and European Standard Population, was 8.3 in 1 million per year ${ }^{3)}$.

MRI usually reveals a well-circumscribed tumor that enhances intensely with the administration of a contrast medium such as gadolinium ${ }^{1,4)}$. Pilocytic astrocytomas usually appear on computed tomography scans as round or oval lesions that are well-defined, iso-dense or slightly hypo-dense, and markedly enhance with the use of a contrast medium. On MRI, pilocytic astrocytomas are typically hypo- or iso-intense on T1 sequences and hyperintense on T2-weighted or fluid-attenuated inversion recovery images. They are typically strongly and diffusely enhanced. These lesions may either contain cysts or consist of a tumor nodule in a cyst (the latter being particularly common for cerebellar and hemispheric tumors) $)^{5,9)}$.

In contrast to ependymomas, pilocytic astrocytomas usually have poorly defined margins, show a more eccentric localization within the cord, and display a patchy enhancement after contrast medium administration. Additional localized hemorrhaging is uncommon and manifests as the so-called "cap sign"15).

Many asymptomatic primary tumors of the spinal cord, especially Grade I meningiomas and peripheral nerve sheath tumors, follow a benign course and can be managed by close observation without surgical or medical intervention. However, surgical intervention is preferred if the tumor is symptomatic. If the lesion is radiographically well defined, the goal of surgical intervention is safe resection and curative treatment. Lesions that may appear well defined on imaging include the ependymoma, WHO Grade I astrocytoma, hemangioblastoma, schwannoma, and the WHO Grade I meningioma ${ }^{7,16,20,21)}$.

Gross total resection of tumors classified as astrocytomas of WHO Grade II or higher is difficult due to the infiltrative nature and poorly defined margins. There are 202 patients with intramedullary tumors.

Over $80 \%$ of WHO Grade I astrocytomas were totally resected surgically, while WHO tumors of Grade II and above had only $12 \%$ total resection rate ${ }^{14)}$. It is therefore accepted that gross total resection of primary spinal tumors should be done whenever possible in order to maximize safe resection for survival $^{2)}$.

Radiation therapy is not recommended as a primary treatment option because of the limited response shown in most cases. Radiation therapy success rates can be influenced by unknown histology if biopsy was not done and low radiation tolerance of spinal cord. In most cases, total excision of tumors is not required because of the low recurrence rates.
A study with a large retrospective analysis including over 1,700 patients with primary spinal gliomas showed an association between radiation therapy and overall survival. A selective bias existed as patients with more severe symptoms would be recommended to receive radiation therapy ${ }^{10)}$. The adjuvant radiation therapy was considered if incomplete excision was achieved or the biopsy results were unclear. In these cases, the use of postoperative radiation therapy was associated with a significantly better survival rate when used to treat the more aggressive infiltrative astrocytomas ${ }^{11)}$.

This research was the motivation for the use of immediate post-biopsy radiation therapy in the case presented in this report. In cases of low WHO grade astrocytomas, however, immediate post-biopsy radiation therapy may be unnecessary. In these cases, the prognostically favorable natural history of the pilocytic astrocytomas suggests that a "watchful waiting" approach would be more advisable. Radiation therapy should therefore only be used if definite clinical or radiographic progression has occurred $^{11)}$. Thus, radiation therapy should be done only in patients who have high grade, infiltrative histological feature, and recurrence following surgical resection of the lesion.
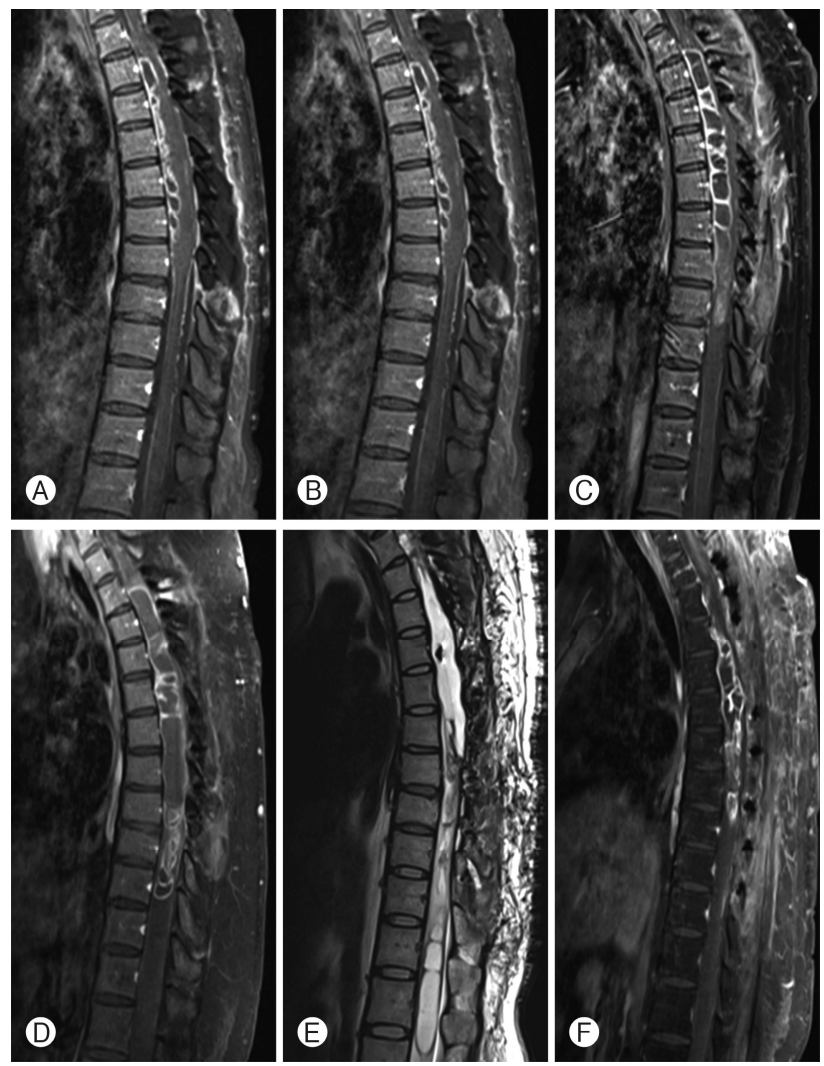

Fig. 3. (A-F) These are serial magnetic resonance imaging after first surgical intervention. The tumor size has increased with time and the patient's neurological symptoms have deteriorated. (E) It is after massive lesionectomy with laminoplasty. 
There are several cases of pilocytic astrocytoma recurrence and associated treatment options. In some cases, partial removal of the tumor was performed followed by $60 \mathrm{~Gy}$ local radiation therapy and chemotherapy. In some other case, decompressive laminoplasty with local radiation therapy showed favorable results.

Follow-up should be considered within 10 years following surgery or adjuvant therapy. This is because new progression in a patient's disease after 10 years of no recurrence is very rare. Routine follow-up beyond 10 years for asymptomatic patients is therefore most likely unnecessary ${ }^{12,13,17,18)}$.

In this case report, the patient's pathology results indicated a WHO Grade I pilocytic astrocytoma which was known for its benign rather than malignant nature. Despite this fact, her neurological deterioration was very aggressive and rapid. Because of the patient' $\mathrm{s}$ age and overall prognosis, an aggressive surgical intervention was determined to be the best course of treatment. At the patient's 1-year follow-up at the outpatient department, the tumor size was decreased compared to previous post-operative MRI findings, proving a successful outcome as a result of the treatment plan (Fig. 3).

\section{CONFLICTS OF INTEREST}

No potential conflict of interest relevant to this article was reported.

\section{REFERENCES}

1. Abel TJ, Chowdhary A, Thapa M, Rutledge JC, Geyer JR, Ojemann J, et al.: Spinal cord pilocytic astrocytoma with leptomeningeal dissemination to the brain. Case report and review of the literature. J Neurosurg 105:508-514, 2006

2. Beneš V, 3rd, Barsa P, Beneš V, Jr., Suchomel P: Prognostic factors in intramedullary astrocytomas: a literature review. Eur Spine J 18:1397-1422, 2009

3. Burkhard C, Di Patre PL, Schüler D, Schüler G, Yaşargil MG, Yonekawa Y, et al.: A population-based study of the incidence and survival rates in patients with pilocytic astrocytoma. J Neurosurg 98:1170-1174, 2003

4. Coakley KJ, Huston J, 3rd, Scheithauer BW, Forbes G, Kelly PJ: Pilocytic astrocytomas: well-demarcated magnetic resonance appearance despite frequent infiltration histologically. Mayo Clin Proc 70:747-751, 1995

5. Collins VP, Jones DT, Giannini C: Pilocytic astrocytoma: pathology, molecular mechanisms and markers. Acta Neuropathol 129:775-788, 2015

6. Fernandez C, Figarella-Branger D, Girard N, Bouvier-Labit C,
Gouvernet J, Paz Paredes A, et al.: Pilocytic astrocytomas in children: prognostic factors--a retrospective study of 80 cases. Neurosurgery 53:544-553, 2003

7. Gezen F, Kahraman S, Canakci Z, Bedük A: Review of 36 cases of spinal cord meningioma. Spine (Phila Pa 1976) 25:727-731, 2000

8. Jiang Y, Lv L, Yin S, Zhou P, Jiang S: Primary spinal pilocytic astrocytoma: clinical study with long-term follow-up in 16 patients and a literature review. Neurosurg Rev 43:719-727, 2020

9. Lee YY, Van Tassel P, Bruner JM, Moser RP, Share JC: Juvenile pilocytic astrocytomas: CT and MR characteristics. AJR Am J Roentgenol 152:1263-1270, 1989

10. Milano MT, Johnson MD, Sul J, Mohile NA, Korones DN, Okunieff P, et al.: Primary spinal cord glioma: a Surveillance, Epidemiology, and End Results database study. J Neurooncol 98:83-92, 2010

11. Minehan KJ, Brown PD, Scheithauer BW, Krauss WE, Wright MP: Prognosis and treatment of spinal cord astrocytoma. Int J Radiat Oncol Biol Phys 73:727-733, 2009

12. Mishima K, Nakamura M, Nakamura H, Nakamura O, Funata $\mathrm{N}$, Shitara N: Leptomeningeal dissemination of cerebellar pilocytic astrocytoma. Case report. J Neurosurg 77:788-791, 1992

13. Obana WG, Cogen PH, Davis RL, Edwards MS: Metastatic juvenile pilocytic astrocytoma. Case report. J Neurosurg 75:972975, 1991

14. Raco A, Esposito V, Lenzi J, Piccirilli M, Delfini R, Cantore G: Long-term follow-up of intramedullary spinal cord tumors: a series of 202 cases. Neurosurgery 56:972-981; discussion 972981, 2005

15. Schittenhelm J, Ebner FH, Tatagiba M, Wolff $M$, Nägele T, Meyermann R, et al.: Holocord pilocytic astrocytoma--case report and review of the literature. Clin Neurol Neurosurg 111: 203-207, 2009

16. Solero CL, Fornari M, Giombini S, Lasio G, Oliveri G, Cimino C, et al.: Spinal meningiomas: review of 174 operated cases. Neurosurgery 25:153-160, 1989

17. Stüer C, Vilz B, Majores M, Becker A, Schramm J, Simon M: Frequent recurrence and progression in pilocytic astrocytoma in adults. Cancer 110:2799-2808, 2007

18. Tamura M, Zama A, Kurihara H, Fujimaki H, Imai H, Kano T, et al.: Management of recurrent pilocytic astrocytoma with leptomeningeal dissemination in childhood. Childs Nerv Syst 14:617-622, 1998

19. Traul DE, Shaffrey ME, Schiff D: Part I: spinal-cord neoplasmsintradural neoplasms. Lancet Oncol 8:35-45, 2007

20. Volpp PB, Han K, Kagan AR, Tome M: Outcomes in treatment for intradural spinal cord ependymomas. Int J Radiat Oncol Biol Phys 69:1199-1204, 2007

21. Yang S, Yang X, Hong G: Surgical treatment of one hundred seventy-four intramedullary spinal cord tumors. Spine (Phila Pa 1976) 34:2705-2710, 2009 